\title{
Aspectos que Compõem o Perfil dos Profissionais Médicos da Estratégia Saúde da Família: o Caso de um Município Polo de Minas Gerais
}

\author{
Aspects of the Family Health Care \\ Professionals' Profile: the Case of a Municipal \\ Hub in Minas Gerais
}

\author{
Simone de Pinho Barbosal \\ Karina Aza Coelho \\ Lorena Miranda de Carvalho ${ }^{I \odot}$ \\ Bianca Sarria ${ }^{1 \oplus}$ \\ Regina Consolação dos Santos ${ }^{I \amalg}$ \\ Ricardo Bezerra Cavalcante ${ }^{I}$
}

\section{PALAVRAS-CHAVE \\ - Atenção Primária à Saúde. \\ - Saúde da Família. \\ - Médico. \\ - Recursos Humanos.}

I Universidade Federal de Juiz de Fora, Governador Valadares, Minas Gerais, Brasil.

II Universidade de Itaúna, Itaúna, Minas Gerais, Brasil. 


\section{KEY-WORDS}

- Primary Healthcare.

- Family Healthcare.

- Physician.

- Human Resources.

Aceito em: 20/6/19

\section{Recebido em: 18/5/19}

ABSTRACT

This study is an exploratory, mixed methods study aimed at identifying the profile of family health care physicians in the municipality of Governador Valadares, Minas Gerais, Brazil, as well as his/ her training and work characteristics. We conducted semi-structured interviews with 36 physicians from family health care teams, using simple descriptive statistics and content analysis in a thematiccategorical mode to organize and analyze the data. The results indicated a predominance of female, Brazilian professionals aged between 24 and 35 years, trained in public institutions and specialized in the field of Family Medicine. Most interviewees belong to the Mais Médicos Program, and have remained in the same team for up to 3 years. The prominent advantages were the identification of the professionals with the work proposal and professional valuation, while the disadvantages were the unstable employment relationship, disregard by management, workplace of difficult access, and excessive demands imposed on them. The facilitating points were teamwork and good relationship with the community, while the most challenging points were the lack of human resources, equipment, finances, and technology. We highlight the importance of the attention given by management to the difficulties to adapt the infrastructure of the Health Care Units and provide a work that contributes to change the health care of the population in this region. Therefore, it is necessary to rethink how physicians can be encouraged to remain in the Family Health Care Strategy, providing them with enhanced job security and prospects. However, it is worth underlining the importance of the profile of family doctors, whose training often diverges from the National Curricular Directives for medical training. These differences make it challenging to solve the issues indicated in this study due to the lack of knowledge about the role of primary healthcare, the importance of integration of the health care network, and lack of knowledge about the physician's attributes at this level of care.

\section{INTRODUÇÃO}

Pensar a atenção primária de um município remete à Política Nacional de Atenção Básica (PNAB), que reafirma a Estratégia Saúde da Família (ESF) como um modelo estratégico para a expansão e consolidação da atenção básica, com forte capacidade de reorientação das práticas de saúde e com impactos relevantes nos indicadores de qualidade de vida, na integralidade das ações e na resolubilidade dos $\operatorname{casos}^{1}$. Isto exige profissionais habilitados e capacitados para atuação específica na área de Saúde da Família, o que, por inúmeros motivos, tem configurado um grande embate na operacionalização e avanço de tal estratégia².

A problemática referente aos recursos humanos atinge amplitude mundial, seja por falta quantitativa de profissionais, seja por déficit qualitativo da oferta do cuidado ou por condições precárias de trabalho ${ }^{2}$. Não há como conceber a viabilização de novos modelos de saúde se não priorizarmos os recursos humanos nesse processo de mudança, com reorientação e fortalecimento da estratégia de formação dos profissionais. Entretanto, a quantidade de profissionais, por si só, não produz efeitos diretos se estes não estão sensibilizados quanto à proposta de trabalho e não conseguem, de fato, causar grandes impactos sobre as reais necessidades da comunidade ${ }^{2,3}$.

É imprescindível que os profissionais atuantes em equipes de Saúde da Família desenvolvam especificidades, tais como: a capacidade de trabalhar em equipe, participando do processo de territorialização e mapeamento da área de atuação; a capacidade de identificar os aspectos determinantes sociais da saúde da população; o conhecimento das famílias e dos grupos da área adscrita, cuidando da população no âmbito tanto da unidade de saúde quanto do domicílio. Além disso, devem-se realizar ações de atenção à saúde de acordo com o perfil populacional, em busca da integralidade por meio de ações de promoção, proteção e recuperação da saúde ${ }^{1,4}$.

Nessa linha de pensamento, a priori, o médico é o foco da pesquisa, por exercer papel de fundamental importância na efetivação dessa modelagem. Nessa perspectiva, é necessário extrair os verdadeiros aspectos e as nuances relacionados ao processo de trabalho desses profissionais que atuam na ESF. É papel do médico realizar atenção à saúde aos usuários cadas- 
trados e, portanto, sob sua responsabilidade; realizar consultas clínicas, procedimentos e atividades em grupo; realizar ações em saúde de acordo com a demanda da comunidade; encaminhar usuários a diferentes níveis de atenção, respeitando referência e contrarreferência e garantindo o acompanhamento do plano terapêutico; colaborar para a educação permanente dos demais profissionais da equipe; e, ainda, participar do gerenciamento de insumos 5 .

Entretanto, para alcançar essas ações, é preciso romper com o modelo hegemônico de formação, pautado na abordagem biologicista, medicalizante e centrado em procedimentos. Nesse modelo pedagógico, o ensino é fragmentado, centrado em conteúdos, desagregando área básica e área clínica, e com aprendizagem prática focada no nível secundário de atenção. Dessa forma, perpetua-se o modelo tradicional de saúde, incentivando-se um padrão técnico de aprendizado, com especialização cada vez mais precoce ${ }^{1,6}$.

Com o estabelecimento da Lei Orgânica do Sistema Único de Saúde $n^{\circ} 8.080 / 90$ e com a posterior definição do foco assistencial na atenção primária para a reestruturação do sistema ${ }^{1}$, o modelo pedagógico tradicional foi amplamente questionado. Com o objetivo de reestruturar a formação médica, o Ministério da Saúde instituiu, em 2001, as Diretrizes Curriculares Nacionais (DCN), que buscam definir uma formação geral humanista, crítica, reflexiva e ética, preparando o futuro graduado para enfrentar os desafios da transformação profissional e das condições de trabalho baseadas numa formação básica sólida e capaz de articular conhecimentos e habilidades para promover a saúde pública ${ }^{7}$.

Tal articulação define-se pela capacidade profissional de autonomia e discernimento perante situações de atenção, gestão e educação em saúde e, ainda, pela capacidade de promover ações de saúde com responsabilidade social e compromisso com os princípios do SUS, sempre em busca da assistência integral à saúde do indivíduo ${ }^{8}$. Em 2014, o Conselho Nacional de Educação, diante dos novos contornos e demandas da área da saúde no Brasil, considerou os novos significados do perfil de competência do médico a ser formado, analisando sua aplicação, distintas abordagens e implicações no campo de formação de trabalhadores de saúde e, então, instituiu as atuais DCN dos cursos de Medicina9

No entanto, existem ainda muitos desafios frente ao estabelecimento de uma identidade médica suficientemente capaz de promover a consolidação das ESF no Brasil. Este artigo teve como objetivo identificar o perfil de profissionais em atuação na atenção primária à saúde, bem como sua formação e características do seu trabalho, entre elas o alinhamento de sua atuação com as reais necessidades da população, tais quais: vín- culo, interdisciplinaridade, trabalho em equipe e preocupação social, e o comprometimento com a construção e consolidação do Sistema Único de Saúde (SUS).

\section{METODOLOGIA}

Trata-se de um estudo de abordagem quali-quantitativa. Justifica-se o uso da abordagem qualitativa pela necessidade de captar o fenômeno que se constrói na vivência dos sujeitos, suas percepções e subjetividades. Três momentos importantes devem ser lembrados na pesquisa quando se opta por estudo qualitativo: a fase de exploração, a investigação e a análise dos resultados finais para elaboração do texto final ${ }^{10,11}$.

O cenário de estudo foi o município de Governador Valadares (MG), que possui 70,11\% de cobertura populacional pela Estratégia Saúde da Família, com 57 equipes. Assim, foram convidados para participar do estudo todos os 57 médicos, o que corresponde a $100 \%$ dos profissionais dessa categoria inseridos nas Equipes de Saúde da Família do município em estudo. Foram excluídos os médicos do Programa de Residência de Medicina de Família e Comunidade (RMFC) e dos distritos sanitários com localização superior a dez quilômetros do aglomerado urbano desse município.

Utilizaram-se entrevistas com roteiro semiestruturado, realizadas em dia e horário agendados com os participantes em local definido por eles. O período de coleta foi de julho a dezembro de 2015. Os entrevistados foram codificados a fim de se preservar sua identidade. Os dados foram tabulados em planilhas e tabelas conforme consensos e diferenças encontrados. A partir de então, foi elaborada uma análise descritiva simples, com discussão pautada na literatura especializada sobre o tema. Na análise dos discursos relacionados às questões dissertativas contidas no questionário, foi empregada a técnica de análise de conteúdo, modalidade temático-categorial ${ }^{12}$.

A pesquisa obedeceu à Resolução 466/2012 do Conselho Nacional de Saúde e foi aprovada pelo Comitê de Ética em Pesquisa com Seres Humanos da Universidade Federal de Juiz de Fora, sob o Parecer consubstanciado de n⿳⺈ 998319. Os participantes manifestaram anuência com a pesquisa mediante assinatura do Termo de Consentimento Livre e Esclarecido (TCLE).

\section{RESULTADOS E DISCUSSÃO}

Dos 57 médicos cadastrados e inseridos na Estratégia Saúde da Família do município em tela, 36 foram entrevistados, e os demais 21 foram excluídos da seguinte forma: quatro eram do Programa de Residência em Medicina de Família e Comunidade (RMFC); no momento da coleta de dados, uma equipe se encontrava incompleta sem o médico; outras duas equipes 
tinham profissionais de férias; quatro médicos se negaram a participar do estudo; três estavam em licença médica; os outros sete estavam localizados em distritos pertencentes ao município, porém com distâncias superiores a dez quilômetros, o que tornava inviável o deslocamento para a coleta de dados.

Em relação aos dados sociodemográficos, observou-se que, do total dos 36 entrevistados, 22 (61,11\%) apresentaram idades entre 24 e 35 anos, e 14 (38,89\%) entre 36 e 68 anos. Vinte e dois eram do sexo feminino e 14 do sexo masculino. Desse conjunto de médicos, 22 (61,11\%) possuem nacionalidade brasileira, e 14 (38,89\%) cubana. Quanto à naturalidade dos brasileiros, dos 21, 19 são da Região Sudeste - 15 de Minas Gerais, três do Espírito Santo e um do Rio Janeiro. Os outros três são de estados variados (Pernambuco, Rio Grande do Sul e Bahia). Cabe salientar que, dos 15 mineiros, 10 são de Governador Valadares (Tabela 1 ).

\begin{tabular}{|c|c|c|}
\hline \multicolumn{3}{|c|}{$\begin{array}{c}\text { TABELA } 1 \\
\text { Características sociodemográficas de médicos } \\
\text { da Estratégia Saúde da Família, município } \\
\text { de Governador Valadares, } 2015\end{array}$} \\
\hline Variáveis & $\mathbf{N}$ & $\%$ \\
\hline \multicolumn{3}{|l|}{ Idade } \\
\hline 24 a 35 anos & 22 & 61,11 \\
\hline 36 a 46 anos & 7 & 19,44 \\
\hline 47 a 57 anos & 5 & 13,89 \\
\hline$>58$ anos & 2 & 5,56 \\
\hline \multicolumn{3}{|l|}{ Sexo } \\
\hline Feminino & 22 & 61,11 \\
\hline Masculino & 14 & 38,89 \\
\hline \multicolumn{3}{|l|}{ Nacionalidade } \\
\hline Brasil & 21 & 61,11 \\
\hline Cuba & 15 & 38,89 \\
\hline \multicolumn{3}{|l|}{ Naturalidade } \\
\hline Região Sudeste & 19 & 90,47 \\
\hline Outros estados & 3 & 9,53 \\
\hline
\end{tabular}

Observou-se que o perfil da amostra converge com o perfil geral de médicos brasileiros, com predominância de profissionais jovens, com idade de até 39 anos e do sexo feminino ${ }^{13}$. Em relação ao predomínio de profissionais do sexo feminino, o dado está em conformidade com os estudos de Silva e Tavares ${ }^{14}$ e Scheffer e Cassenote ${ }^{15}$, que mencionam uma feminização da saúde. Esse fato está muito relacionado com a maior inserção das mulheres no ensino superior e no mercado de trabalho em geral ${ }^{16}$. Em 2009, as mulheres passaram a ser a maioria a concluir o curso de Medicina no Brasil, e a partir desse ano essa tendência só cresceu. O cenário só difere entre os médicos mais idosos, entre os quais ainda predomina o sexo masculino. É importante salientar que a ascendência feminina da profissão se reflete na escolha da especialização médica. As áreas de cirurgia e urgência e emergência são de maior preferência entre os profissionais do sexo masculino, o que sinaliza a contento a preferência das mulheres por especializações relacionadas ao cuidado primário, como Medicina de Família e Comunidade e Pediatria ${ }^{15}$.

Em relação ao tempo de formação, a maioria dos profissionais apresenta um tempo relativamente baixo, de um a cinco anos. Isto pode ser explicado pelo fato de que muitos médicos recém-formados, em busca de experiência, optam por buscar uma oportunidade de trabalho no serviço público - no caso, junto à Estratégia Saúde da Família' ${ }^{17}$ (Tabela 2).

TABELA 2

Características relacionadas à formação profissional dos médicos da Estratégia Saúde da Família do município de Governador Valadares, 2015

$\begin{array}{lrr}\text { Variáveis } & \text { N } & \% \\ \text { Tempo de formação (anos) } & & \\ 1 \text { a } 5 & 13 & 36,11 \\ 6 \text { a } 10 & 8 & 22,22 \\ 11 \text { a } 15 & 4 & 11,11 \\ 16 \text { a } 20 & 2 & 5,56 \\ \text { > ou = 20 } & 9 & 25,00 \\ \text { Instituição formadora } & & \\ \text { Pública } & 21 & 58,33 \\ \text { Privada } & 15 & 41,67 \\ \text { Local de formação } & & \\ \text { Brasil } & 20 & 55,56 \\ \text { Cuba } & 16 & 44,44\end{array}$

Segundo o censo de educação superior ${ }^{18}$, em relação aos cursos de graduação presenciais, há no Brasil 2,6 alunos matriculados em instituições privadas para cada aluno matriculado na rede pública, o que indica que os discentes, de modo geral, estão mais concentrados em instituições privadas de ensino. No que tange à distribuição dos cursos de Medicina nessas instituições, também se observa um predomínio das instituições privadas. Do total de 197 cursos, 114 são privados e 83 são públicos. Contudo, o número de profissionais que apresentaram formação em instituições de ensino públicas correspondeu à maioria da amostra. Esses dados vão ao encontro de outra pesquisa sobre o perfil de profissionais da Estratégia Saúde da Família, no Estado de Mato Grosso ${ }^{19}$, na qual a maioria dos profissionais $(61,3 \%)$ tinha graduação também em instituições públicas.

Observa-se, também, um número considerável de médicos que tiveram sua formação em Cuba atuando na ESF no município devido ao Programa Mais Médicos, criado em 2013 pelo governo federal com o objetivo principal de melhorar a 
qualidade do atendimento aos usuários do SUS e que atraiu médicos estrangeiros para regiões brasileiras onde há vazios assistenciais e consequente escassez profissional ${ }^{2,20}$. A Tabela 3 apresenta os aspectos formadores mais específicos para a área, como estágio curricular, realização de curso de especialização na área de Medicina de Família e Comunidade ou outra especialidade em áreas afins, como Saúde Coletiva, Saúde Pública e Saúde da Família. Além disso, foi abordada a participação em curso de capacitação e que tipos de capacitação ainda gostariam de realizar.

\begin{tabular}{|c|c|c|}
\hline \multicolumn{3}{|c|}{$\begin{array}{l}\text { TABELA } 3 \\
\text { Características relacionadas à especialidade e } \\
\text { ao perfil de formação profissional, } 2015\end{array}$} \\
\hline Variáveis & $\mathbf{N}$ & $\%$ \\
\hline \multicolumn{3}{|l|}{ Estágio da área de SF } \\
\hline Sim & 32 & 88,88 \\
\hline Não & 4 & 11,12 \\
\hline \multicolumn{3}{|c|}{ Especialidade na área de MFC ou áreas afins } \\
\hline Sim & 26 & 72,22 \\
\hline Não & 6 & 16,66 \\
\hline Em curso & 4 & 11,12 \\
\hline \multicolumn{3}{|l|}{ Capacitação profissional } \\
\hline Sim & 35 & 97,22 \\
\hline Não & 1 & 2,78 \\
\hline \multicolumn{3}{|l|}{ Modalidade de capacitação } \\
\hline Nenhuma & 4 & 9,31 \\
\hline Curso de aperfeiçoamento & 6 & 13,95 \\
\hline Especialização & 15 & 34,88 \\
\hline Mestrado & 12 & 27,91 \\
\hline Doutorado & 6 & 13,95 \\
\hline
\end{tabular}

* Na variável modalidade de capacitação foi permitida mais de uma resposta.

A Tabela 3 aponta que, do conjunto de médicos da pesquisa, 32 (88,88\%) relataram ter realizado estágio curricular na área de Saúde Pública, e outros 4 (11,12\%) marcaram a opção "não". Sobre a especialização, 26 declararam possuir especialização em Saúde Pública ou em Medicina de Família e Comunidade, enquanto $6(16,22 \%)$ não possuem especialização na área. Os demais profissionais estão com o curso em andamento. Acerca da capacitação profissional, a grande maioria dos profissionais - $35(97,22 \%)$ - referiu ter participado de algum curso ao longo da carreira, contudo, em relação à modalidade de capacitação a que ainda gostariam de ter acesso, 15 optaram por curso de especialização, 12 por mestrado, 6 citaram cursos de aperfeiçoamento com carga horária inferior a 360 horas e 6 mencionaram curso de doutorado. As áreas mais solicitadas para capacitação foram, em ordem decrescente, Saúde da Criança e do Adolescente, do Idoso, do Adulto e da Mulher.
Em razão da identificação com a proposta, muitos médicos escolhem trabalhar na ESF. Isto é observado na amostra, na qual a grande maioria dos profissionais já havia realizado algum estágio na área da Saúde da Família e, portanto, conhece e se interessa por ela. Achado semelhante está nos estudos de Albuquerque e colaboradores ${ }^{22}$, em que a maioria dos médicos escolheu trabalhar na ESF por admirar a proposta. Além disso, as novas diretrizes para o curso de Medicina apresentam disciplinas para inserir os estudantes, desde o início de sua formação, no cenário real de suas práticas.

Tal achado mostra que a gestão realiza majoritariamente a vinculação trabalhista por programas de governo, o que pode dificultar a permanência do profissional por mais tempo nas equipes, prejudicando o estabelecimento de vínculo com a comunidade e a continuidade do cuidado, diretrizes operacionais da ESF. As formas de vínculo trabalhista estabelecidas pela gestão municipal, muitas vezes, têm caráter partidário, baseado em interesses eleitorais e questões políticas. Isto pode se refletir na atuação do profissional da ESF. Desse modo, fica comprometida a autonomia profissional para a realização de intervenções necessárias no território da equipe.

\begin{tabular}{|c|c|c|}
\hline \multicolumn{3}{|c|}{$\begin{array}{l}\text { TABELA } 4 \\
\text { nodalidade de empregabilidade } \\
\text { Estratégia Saúde da Família, } \\
\text { lador Valadares, } 2015\end{array}$} \\
\hline Variáveis & $\mathbf{N}$ & $\%$ \\
\hline \multicolumn{3}{|l|}{ Modalidade de trabalho } \\
\hline Programa Mais Médicos & 30 & 83,33 \\
\hline Convencional & 5 & 13,89 \\
\hline Provab & 1 & 2,78 \\
\hline \multicolumn{3}{|c|}{ Tempo de atuação como MF } \\
\hline 1 a 3 anos & 17 & 45,95 \\
\hline 4 a 6 anos & 5 & 16,22 \\
\hline$>7$ anos & 14 & 37,83 \\
\hline \multicolumn{3}{|c|}{ Permanência na Equipe (ESF) } \\
\hline$<1$ ano & 14 & 35,13 \\
\hline 1 a 3 anos & 19 & 56,76 \\
\hline 4 a 6 anos & 1 & 2,70 \\
\hline$>7$ anos & 2 & 5,41 \\
\hline
\end{tabular}

Além disso, a tabela 4 demonstra que a falta de estabilidade no trabalho dificulta a permanência na $\mathrm{ESF}^{22}$. Por outro lado, embora os concursos públicos possibilitem maior autonomia profissional, pode-se inferir que poucos profissionais médicos estão dispostos a realizá-los, devido à predominância do modelo de formação centrado na especialização e hospitalocêntrico, que limita o entendimento sobre o trabalho na atenção primária e a busca pela medicina da família e comunidade. 
Quanto ao tempo de atuação na equipe, este se relaciona com a variável do tempo de formação. Existe um perfil de médicos recém-formados com curto período de atuação, o que corrobora o estudo que aponta um perfil de recém-egressos de Medicina que costumam, de modo geral, trabalhar em equipes de Saúde da Família como primeira experiência profissional, até serem aprovados em programas de residência médica ${ }^{17}$.

Cabe inferir que essa postura vai ao encontro do tipo de formação ofertada, baseada no modelo flexneriano, com uma linha curricular estruturada na fragmentação do sujeito e, consequentemente, na especialização do conhecimento, como espaço de maior reconhecimento profissional, e na via contrária uma matriz curricular incipiente no que tange à carga horária de disciplinas da área da Saúde Coletiva.

Nota-se que mais de $90 \%$ dos profissionais entrevistados tinham até três anos de permanência na equipe, remetendo ora ao período de vigência estabelecido pelo Programa Mais Médicos, ora à rotatividade profissional que circunda a ESF, conforme aponta o estudo de Galavote e colaboradores ${ }^{21}$ e Magnago e Pierantoni ${ }^{23}$. No que tange à escolha da área de atuação de Medicina de Família e Comunidade, a maioria dos entrevistados a apontou como uma área favorável do ponto de vista da empregabilidade, identificação com a área, satisfação profissional e salarial, e também a primeira oportunidade de trabalho. Resultado semelhante foi encontrado nos estudos de Oliveira e colaboradores ${ }^{2}$, Galavote e colaboradores ${ }^{21}$ e Soratto e colaboradores ${ }^{24}$, que evidenciaram como principal motivação para essa escolha da área a realização profissional e possibilidade de melhor remuneração.

A respeito de experiências anteriores de trabalho na área, 23 (44,23\%) relataram atuação no eixo assistencial, 12 (23,08\%) citaram experiência no eixo da docência, e 5 (9,61\%) médicos no eixo da gestão. Já 10 (19,23\%) expuseram não ter qualquer experiência anterior, tendo em vista o fato de ser o primeiro emprego. Os resultados mostram que alguns profissionais aqueles com maior tempo de formação - tiveram mais de uma experiência anterior na área.

O perfil jovem da amostra condiz com os resultados encontrados sobre a inexperiência anterior na área. Cabe salientar o perfil do médico que teve sua formação em Cuba, onde esses profissionais também são preparados para atuação em áreas de gestão e docência. Pode-se inferir que os médicos com experiências em gestão são capazes de gerir melhor os recursos destinados ao atendimento da população assistida pela equipe, acarretando um impacto positivo na distribuição e otimização financeira do sistema de saúde. Esse perfil vai ao encontro das atribuições do médico definidas na PNAB 20175, em que o profissional deve participar da gerência dos insu- mos junto ao enfermeiro, técnico em enfermagem, cirurgião-dentista e técnico em saúde bucal.

Sobre as vantagens de trabalhar na ESF, a maioria apontou a identificação com a proposta de trabalho, seguida da autonomia e valorização profissional, com algumas opções relacionadas a boa remuneração. Também foram citadas, de forma mais pontual, atuar com a população mais necessitada, como forma de emprego transitório, como oportunidade de atuar na prevenção de doenças e proximidade com o usuário. Um dos entrevistados negou qualquer vantagem em trabalhar na ESF, ponto de inquietude deste estudo que merece reflexão (Quadro 1).

\section{QuAdro 1}

Classificação decrescente das vantagens em atuar na

Estratégia Saúde da Família, Governador Valadares, 2015

Vantagens

Identificação com a proposta

Autonomia profissional

Valorização profissional

Boa remuneração

Não considero vantagem atuar na ESF

Atuar com população mais necessitada

Emprego transitório

Atuar na prevenção de doenças

Proximidade com o usuário

A Estratégia Saúde da Família é a principal proposta do governo brasileiro para a tentativa de reformular a atenção primária em saúde (APS) e fortalecer o SUS PNAB, 20175. Porém, para a estratégia atingir esse objetivo, é essencial um profissional com atribuições adequadas e que se identifique com a proposta do programa. Logo, a identificação com a proposta da ESF é o maior fator de fixação dos médicos nas unidades de saúde. $\mathrm{O}$ fato de a atenção primária atuar mais próxima da comunidade, visando à prevenção e promoção da saúde por meio da criação de um vínculo entre esta e os profissionais da saúde, e responder de forma mais efetiva aos anseios da população também tem grande influência na escolha dos médicos que se identificam com o programa ${ }^{25}$.

Segundo Albuquerque e colaboradores ${ }^{22}$, a motivação é um fator importante para a produtividade do profissional, visto que este, motivado e satisfeito, se apresenta mais disposto a desempenhar suas funções com excelência. Nesse contexto, é importante identificar os fatores que levam à motivação dos trabalhadores. A autonomia é um item de motivação: significa dizer que o profissional tem liberdade para desenvolver sua atividade, de acordo com os conhecimentos teóricos aprendidos em sua formação. Segundo Galavote e colaboradore ${ }^{21}$, profissionais que trabalham em ambientes mais democráticos e são estimulados 
a desenvolver diversas funções sentem-se mais responsáveis e conseguem enfrentar melhor as dificuldades no trabalho.

Outra vantagem apontada foi a valorização profissional, fator importante para a motivação e que poderia contribuir para a redução da rotatividade dos médicos nas equipes de ESF. Segundo, Scheffer e Cassenote ${ }^{15}$, a adoção de medidas de valorização profissional poderia aumentar a presença, disponibilidade e dedicação exclusiva dos médicos no serviço público.

Cabe salientar que o médico é o principal agente para que a Estratégia Saúde da Família se concretize. Assim, é essencial a formação de profissionais capacitados, satisfeitos e motivados com o trabalho. Quando o estudante não possui, desde a graduação, a formação voltada para a atuação na ESF, o trabalho se torna monótono, e o trabalhador, insatisfeito ${ }^{17}$. Assim, pode-se inferir que ainda há médicos atuando na Estratégia Saúde da Família sem o perfil profissional por ela demandado, e, por esse motivo, esse médico talvez esteja se sentindo desmotivado, o que ocasiona insatisfação com a proposta. A falta desse perfil pode ser reflexo de uma formação mais tradicional, no molde tecnicista e biologicista. Essa realidade é semelhante à dos estudos de Cruz e colaboradores ${ }^{26}$, que encontraram nos acadêmicos de Medicina uma visão ainda biomédica, pautada na doença, o que vai em direção oposta aos princípios da ESF.

Em relação às desvantagens encontradas em atuar na ESF, a maioria as negou. Os que consideraram algum tipo de desvantagem citaram de forma decrescente: vínculo empregatício instável, desamparo da gestão, locais de difícil atuação, excesso de cobrança. Ainda foram apontadas: carga horária de 40 horas semanais, falta de medicamentos, falta de recursos diversos, dificuldade na integração entre os níveis de atenção primário e secundário, referência e contrarreferência, amplo território adscrito, instabilidade de emprego e baixa resolutividade, segundo exposto no Quadro 2.

É interessante observar que a maioria dos médicos entrevistados não considera desvantajoso atuar na ESF. Dados semelhantes foram compilados por Damno e colaboradores ${ }^{27}$. As principais desvantagens encontradas foram excesso de burocracia, falta de estímulo à educação permanente, desamparo da gestão, falha no processo de referência e contrarreferência, suporte estrutural, além da ampla abrangência de atuação, bem como excesso de demanda.

De acordo com Galavote e colaboradores ${ }^{21}$, a carga horária de trabalho também se mostra um fator de desvantagem apontada pelos profissionais. É interessante contrastar com os dados compilados por Damno e colaboradores ${ }^{27}$, em que os médicos que trabalhavam menos de 40 horas semanais consideravam interessante a Portaria n⿳0 2.488, de 21 de outubro de 2011, que redefine a carga horária dos profissionais médicos
QuAdro 2

Classificação decrescente de desvantagens em atuar na

Estratégia Saúde da Família, Governador Valadares, 2015

Desvantagens

Não considero desvantagem atuar na ESF

Vínculo empregatício instável

Desamparo da gestão

Locais de atuação de difícil acesso

Excesso de cobranças

Carga horária de trabalho (40 horas)

Falta de recursos

Falta de medicamentos

Dificuldade na integração entre o nível de atenção primário e o secundário

Amplo território adscrito

Instabilidade de emprego

Baixa resolutividade

Baixa escolaridade da população adscrita

Desvalorização da atenção primária

na ESF por permitir maior tempo para qualificação e capacitação profissional, assim como aqueles que trabalhavam no período de 40 horas destacaram que tal medida poderia prejudicar a criação de vínculo com a população.

O vínculo empregatício instável gera no profissional insegurança, falta de perspectiva de crescimento profissional e ausência de direitos trabalhistas. Tal realidade culmina em vínculos frágeis com a comunidade e desqualificação no atendimento, assim como contribui para o aumento da rotatividade. Rossoni ${ }^{28}$ mostrou que o vínculo estável incentiva os profissionais médicos a permanecerem no trabalho, visto que se sentem seguros para desenvolver estratégias e planos nas equipes de que participam, bem como se tornam mais motivados no trabalho, o que ocasiona melhorias na ESF ${ }^{22,23}$. Essa relação de trabalho pode ser criada por meio de contratos estatutários e de concursos públicos, que dificilmente são realizados.

Juntamente a esse fator, nota-se o descontentamento com a administração da gestão, visto que dificuldades no gerenciamento do sistema financeiro se refletem diretamente na distribuição dos recursos, ocasionando deficiência de insumos nas unidades e infraestrutura precária. Isto pode estar relacionado com o fato de que muitos gestores municipais não possuem formação em saúde, o que dificulta seu desempenho ${ }^{23}$.

Quanto aos locais de difícil atuação, observa-se que esse é um fator determinante para a rotatividade dos profissionais de saúde, conforme descrito nos estudos de Galavote e colaboradore ${ }^{21}$. Por serem a porta de entrada do sistema e possuírem metas para proporcionar mudança nos territórios, as unidades de saúde devem ser instaladas em regiões estraté- 
gicas, nos diferentes bairros nas cidades. Diante disso, nota-se um confronto de ideais entre o esperado pelos profissionais e a proposta de ampliação do acesso à saúde para todos, definida nas propostas da APS.

É importante salientar que muitos bairros estão localizados em lugares distantes devido ao processo histórico brasileiro, em que houve rápido crescimento das cidades de modo desordenado e inadequado. Pode-se entender que o excesso de cobranças referido se deve à grande demanda de consultas e responsabilidades, originária da persistência do modelo de atendimento centrado na doença que se une aos esforços para concretizar as propostas do trabalho na ESF, de prevenção e promoção em saúde ${ }^{29}$.

A integração da rede e os níveis de atenção são dificuldades enfatizadas. Galavote e colaboradores ${ }^{21}$ atribuem esse fato à grande rotatividade, à escassez de médicos com perfil generalista na atenção primária e à persistência do modelo médico centrado na doença. $\mathrm{O}$ perfil do médico de formação centrada na doença ocasiona maior número de encaminhamentos para a atenção secundária, o que contraria a proposta de resolutividade da APS. Desse modo, ressalta-se a Diretriz Curricular Nacional de $2014^{\circ}$, que traz ao ensino a necessidade da formação do médico com perfil generalista. Além disso, destaca-se a importância da centralização da ESF para referência e contrarreferência do usuário e a continuidade do cuidado.

Outro ponto levantado foi o amplo território para o atendimento, indo ao encontro dos resultados achados por Albuquerque e colaboradores ${ }^{22}$ - de insatisfação dos médicos em relação a esse assunto -, contrapondo a regulamentação da $\mathrm{PNAB}^{5}$, em que cada equipe de Saúde da Família deve se responsabilizar por no máximo 4 mil pessoas, de modo que a equipe possa conhecer todos os aspectos que envolvem a comunidade.

Diante de tudo, o profissional ressalta como desvantagem a baixa resolutividade, devido à problemática que envolve a efetivação da teoria na prática. Além disso, cabe ressaltar a interferência da formação do perfil médico, ainda centrada no indivíduo e na especialidade, o que acarreta entraves para a resolutividade.

\section{CONSIDERAÇÕES FINAIS}

Este estudo aponta um perfil de médicos jovens, recém-formados, em sua maioria do sexo feminino, brasileiros e com formação em instituições públicas de ensino. Quanto à formação profissional, observam-se médicos capacitados e que já tinham algum contato prévio com a área, como, por exemplo, estágio na área de Saúde da Família e especialização em Medicina da Família e Comunidade. Diante disso, nota-se a aproximação das características almejadas para o médico da ESF.
Salienta-se a importância da atenção da gestão para as dificuldades ressaltadas, de modo a adequar a infraestrutura das unidades de saúde para proporcionar um trabalho que colabore com a mudança da saúde da população do território adscrito. Além disso, é necessário repensar a maneira de incentivar o médico a permanecer na ESF, proporcionando-lhe segurança e perspectivas no trabalho.

Acerca das desvantagens mencionadas neste estudo, cabe ressaltar que fatores políticos e econômicos influenciam diretamente a preparação e o conhecimento da gestão em relação à relevância da ESF, bem como o seu objetivo de reorganização do modelo de saúde, o que, por vezes, pode dificultar a efetividade da política pública de saúde vigente. Além disso, cabe salientar a importância do perfil do médico, que, muitas vezes, teve uma formação diferente da preconizada nas Diretrizes Curriculares Nacionais do Curso de Medicina, dificultando a resolução dos problemas apontados neste estudo, haja vista o desconhecimento do papel da atenção primária, da importância da integração da rede de saúde e dos atributos do médico nesse nível de atenção.

\section{REFERÊNCIAS}

1. Fertonani HP, Pires DEP, Biff M, Scherer MDA. Modelo assistencial em saúde: conceitos e desafios para a atenção básica brasileira. Ciênc Saúde Coletiva 2015;20(6)1869-78.

2. Oliveira MPR, Carvalho IH, Menezes FA, Sousa LM, Peixoto MRG. Formação e qualificação de profissionais de saúde: fatores associados à qualidade da atenção primária. RevBrasEducMéd 2016;40(4)547-59.

3. Merhy EE. Em busca da qualidade dos serviços de saúde: os serviços e modelo porta aberta para a saúde e o modelo tecno-assistencial em defesa da vida. In: Cecilio LCO. Inventando a mudança na saúde. 3. ed. São Paulo: Hucitec; 2006. p.117-60.

4. Scherer MDA, Pires DEP, Jean R. A construção da interdisciplinaridade no trabalho da Equipe de Saúde da Família. CiencSaude Coletiva 2013;18(11)3203-12.

5. Brasil, Ministério da Saúde. Política Nacional de Atenção Básica - PNAB. Brasília: Ministério da Saúde;2017.

6. Ceccim RB, Feuerwerker LCM. Mudança na graduação das profissões de saúde sob o eixo da integralidade. Cad Saúde Pública 2004;20(5):1400-10.

7. Gomes TLCS, Higa EFR, Passos AHR, Soares MOM, Otani MAP, Souto BGA. Integralidade na Atenção Primária à Saúde: compreensão dos estudantes de medicina e enfermagem. Atas do 6. Congresso Ibero-Americano em Investigação Qualitativa em Educação; 2017 jul. 12-14; Salamanca, Espanha. Salamanca: CIAIQ;2017. 
8. Gomes TLCS, Higa EFR, Passos AHR, Soares MOM, Otani MAP, Souto BGA. A visão de estudantes de medicina e enfermagem sobre a integralidade na Atenção Primária à Saúde. RevPsi Divers Saúde 2018;7(1)112-9.

9. Brasil, Ministério da Educação. Resolução CNE/CES no 3 de 20 de junho de 2014. Institui Diretrizes Curriculares Nacionais do Curso de Graduação em Medicina e dá outras providências. Diário Oficial da União. Brasília, 23 jun. 2014; Seção 1, p.08/11.

10. Minayo MC. Amostragem e saturação em pesquisa qualitativa: consensos e controvérsia. RevPesqQualit 2017;5(7):1-12.

11. Yin RK. Estudo de caso: planejamento e métodos. 4. ed. Porto Alegre: Bookman;2015.

12. Bardin L. Análise de conteúdo. 4.ed. Lisboa:Ed. 70;2015.

13. Conselho Federal de Medicina. Demografia médica no Brasil 2015. São Paulo: Conselho Federal de Medicina do Estado de São Paulo;2015.

14. Silva DAJ, Tavares MFL. Ação intersetorial: potencialidades edificuldades do trabalho em equipes da Estratégia Saúde da Família na cidade do Riode Janeiro. Saúde Debate 2016;40(111)193-205.

15. Scheffer MC, Cassenote AJF. A feminização da medicina no Brasil. RevBioét 2013;21(2)268-77.

16. Ricoldi A, Artes A. Mulheres no ensino superior brasileiro: espaço garantido e novos desafios. ExÆquo 2016;(33)149-61.

17. Araújo CA, Michelotti FC, Ramos TKS. Government provision programs: profile and motivations of physicians who migrated from the Primary Care Professional Valorization Program (Provab) to the More Doctors Program in 2016. Interface 2017;21(supl. 1)1217-28.

18. Brasil, Ministério da Educação. Censo da Educação Superior 2014 - Notas Estatísticas. Brasília: INEP;2014.

19. Canesqui AM, Spinelli MAS. Saúde da família no Estado de Mato Grosso, Brasil: perfis e julgamentos dos médicos e enfermeiros. Cad Saúde Pública2006;22(9)1881-92.

20. Pinto HA, Sales MJT, Oliveira, FP, Brizolara R, Figueiredo AM, Santos JT. O Programa Mais Médico e o fortalecimento da Atenção Básica. Divulg Saúde Debate 2014;(51)105-120.

21. Galavote HS, Franco TB, Freitas PSS, Lima EEA, Garcia $\mathrm{ACP}$, Andrade MAC, et al. A gestão do trabalho na estratégia saúde da família: (des)potencialidades no cotidiano do trabalho em saúde. Saúde Soc 2016;25(4)988-1002.

22. Albuquerque GC, Murakami VYC, Taveira BLS, Carvalho ILF, Lima RL, Marques ICS. Satisfaction of physicians working in Primary Health Care Guilherme Souza. Rev APS 2017;20(2)221-30.
23. Magnago C, Pierantoni CR. Dificuldades e estratégia de enfrentamento referentes à gestão do trabalho na Estratégia Saúde da Família, na perspectiva dos gestores locais: a experiência dos municípios do Rio de Janeiro (RJ) e Duque de Caxias (RJ). Saúde Debate 2015;39(104)9-17.

24. Soratto J, Fernandes SC, Martins CF, Tomasi CD, Zanini MTB, Fertonani HP. Aspectos geradores de satisfação e insatisfação dos profissionais da estratégia saúde da família de um município de pequeno porte da região sul do Brasil. Rev CEFAC 2018;20(1)69-78.

25. Issa AHTM, Garcia-Zapata MTA, Rocha AC, Sandré BB, Dutra ACF, Martins ILO, et al. Fatores influenciadores na escolha pela medicina de família segundo estudantes numa região neotropical do Brasil. RESU 2017;5(2)56-65.

26. Cruz AR, Reis KMN, Segheto W, Oliveira AF, França AAP, Alencar JAR, et al. Formação e percepção do profissional médico sobre saúde pública. Rev Científica Fagoc Saúde 2017;2(2)80-8.

27. Damno SH, Moriyama MC, Picoli RP, Sartori BG, Asato SMM, Carvalho GC, et al. Perfil profissional dos médicos atuantes na estratégia Saúde da Família no Município de Campo Grande-MS. Encontro: revpsicol 2013;16(25)125-37.

28. Rossoni E. Residência na atenção básica à saúde em tempos líquidos. Physis 2015;25(3):1011-31.

29. Brito GEG, Mendes ACG, Santos NPM. Purpose of work in the Family Health Strategy. Interface 2018;22(64):77-86.

\section{CONTRIBUIÇÃO DOS AUTORES}

Elaboração do projeto: SPB, KAC, LMC, BS.

Análise formal: SPB, KAC, LMC, BS.

Metodologia: SPB, KAC, LMC, BS.

Coleta de dados: SPB, KAC, LMC, BS.

Escrita - rascunho original: SPB, KAC, LMC, BS.

Escrita - revisão critica final: SPB, KAC, LMC, BS, RBC, RCS.

Análise final do artigo: RBC, RCS.

\section{CONFLITO DE INTERESSES}

Os autores declaram não haver conflito de interesses.

\section{ENDEREÇO PARA CORRESPONDÊNCIA}

Simone de Pinho Barbosa

Universidade Federal de Juiz de Fora - Campus de Governador Valadares

Departamento de Medicina

Rua Manoel Byrro,241sala106 - Vila Bretas

Telefone: (33) 3301.1000 ramal 1585

E-mail: simone.pinho@ufjf.edu.br 